\title{
Mobile application development framework to support farming as a business via benchmarking: the case of Tanzania
}

\author{
John Joel Kyaruzi ${ }^{1 *}$, Zaipuna Obedi Yonah ${ }^{2}$ and Hulda Shaidi Swai ${ }^{3}$ \\ Research Scholar, Nelson Mandela African Institution of Science and Technology (NM-AIST) in Arusha, Tanzania ${ }^{1}$ \\ Senior Researcher, Nelson Mandela African Institution of Science and Technology (NM-AIST) in Arusha, \\ Tanzania $^{2}$ \\ Associate Professor, Nelson Mandela African Institution of Science and Technology (NM-AIST) in Arusha, \\ Tanzania $^{3}$
}

Received: 10-June-2019; Revised: 07-October-2019; Accepted: 10-October-2019

(C)2019 John Joel Kyaruzi et al. This is an open access article distributed under the Creative Commons Attribution (CC BY) License, which permits unrestricted use, distribution, and reproduction in any medium, provided the original work is properly cited.

\begin{abstract}
Contributions from various researchers and scholars have made major advances relevant to a wide range of mobile applications at various scales. Although current agricultural and rural development $(A R D)$ systems have features that are needed for farming as a business (FAAB). It is established that all of them have limitations in realising benchmarking as their basic principle. Common limitations across all systems, include 1) scarcity of data for modelling, evaluating, and applying benchmarking and 2) inadequate knowledge systems that effectively communicate benchmarking results to farmers. These two limitations are greater obstacles to developing useful mobile applications than gaps in conceptual theory or available methods for using "Farming as a Business via Benchmarking (FAABB)". This paper presents reviews of the current state of mobile application development frameworks, focusing on their capabilities and limitations to support FAABB. The paper presents a new framework to support $F A A B B$ in the Tanzanian context, which is implemented through a FAABB cyber studio hosted at the Nelson Mandela-African Institution of Science and Technology (NM-AIST) in Tanzania. The framework promises to address not only the knowledge codification problem, but also the need for a cultural change among agricultural researchers to ensure that data for addressing the range of use-cases are available for future mobile application development. The FAABB framework has been tested in the Southern Agricultural Growth Corridor of Tanzania (SAGCOT) and its initial results provides a useful starting point for developing m-apps for addressing ARD challenges in developing countries.

\section{Keywords}

Agricultural and rural development (ARD), Farming-as-a-business via benchmarking (FAABB), M-apps development frameworks, Use-cases.
\end{abstract}

\section{Introduction}

In many developing countries, agricultural supplyside (i.e. smallholder farmers) is normally weak and cannot meet demands of formal markets [1]. Problems include lack of mechanization; lack of irrigation schemes with too much reliance on rain; lack of skills and knowledge on farming-as-abusiness (FAAB); poor transportation and logistical networks; and lack or poor farm inputs (e.g. seeds, fertilizers, pests, etc.) [2, 3]. In recent years, acceptance of agricultural and rural development (ARD) science has started to address challenges such as efficient production, managing profitability, sustaining the environment.

*Author for correspondence

365
In recent years, acceptance of agricultural and rural development (ARD) science has started to address challenges such as efficient production, managing profitability, sustaining the environment, as well as policy issues to force farming systems meet developing countries' goals. Suitable solutions that address FAAB will likely benefit from technologies that apply codified information and domain knowledge [4].

For the case of Tanzania, in particular, the problem is further aggravated due to insufficient number of experienced extension officers and farm managers engaged in agriculture in general [5] and dairy subsector in particular [6] that can assist smallholder farmers to be responsive to FAAB. For example, 
while extension officers are expected to be the primary backstopping agents for farmers in bridging benchmarking information and knowledge gaps, it has been observed that most extension officers are assigned to subsectors for which their understanding of domain knowledge is handicapped [7]. In some cases, one extension officer serves farmers engaged in multiple subsectors, whose workings is ill informed about [8].

Nelson Mandela-African Institution of Science and Technology (NM-AIST) realised that a cyber-studio is needed to address most of the above problems through a targeted framework for developing mobile applications as tools for realising farming as a business via benchmarking (FAABB). The outcomes of such analyses could be more accessible to farmers through specialised mobile applications (m-apps). Reference [9] testifies that mobile handsets have started penetrating in developing countries' rural areas. Consequently, m-apps can be developed to complement extension officers' roles in supporting FAABB.

The objectives of this work are threefold:

- To define a framework of critical value chain actors, their functions, and optimal orientation that allow for managing the farm's efficiency and profitability through benchmarking.

- To develop a decision support framework that facilitates farmers to conduct guided FAABB electronically.

- To develop the knowledge codification framework to ensure that FAABB data is available for mobile application developers for addressing a wide range of use-cases.

\subsection{Achieving farming as a business through} benchmarking

Gaining from agriculture for smallholders requires strong linkages between farmers and their best markets. Good markets are determined by the prices offered to farm produce and this can be guaranteed if farmers' productivity meet market demands in terms of both quality and quantities demanded by their markets. The productivity factor is therefore the primary benchmarking factor for poverty, elimination of smallholder farmers in rural farming. For example, with farm productivity in Tanzania at just one quarter of the global average [10], there is a huge potential to increase agricultural output, and thereby boost incomes for smallholder farmers and their communities [11].
Farmer groups themselves need to be assisted to increase productivity through technology, quality and quantity enhancements all, primarily, aimed at supporting smallholder farmers [3]. Supporting farmers to increase productivity requires proper choices of farms, administration and monitoring of extension services provided to these farms, and product value additions through postharvest handling. All of these choices and their monitoring are formally achieved through benchmarking [12].

FAABB is therefore concerned with engaging farmers to achieve both productivity and profitability. Improving the performance of FAABB, therefore, requires a better understanding of both the business and technical aspects of farming [11]. At farm-level, 'benchmarking' is conducted by an extension-officer who plays the role of farm manager on behalf of a group of farmers and uses benchmarking techniques to identify problems that prevent the farm from achieving its full value and devise means to improve; hence the concept of FAAB via Benchmarking, in short FAABB.

The term 'benchmarking' used in Farm benchmarking is a type of survey analysis that allows you to compare how your farm stacks up to the operations, revenue and costs expended by other farmers. This analysis gives you a more transparent look at what successful other farmers may be experiencing and how you may be able to reach a similar success of your own farm operations. It can also help to trace the farmers' economic health checks or their qualification for funding.

Despite the good intentions of FAABB, most smallholder farmers' organizations still suffer from the knowledge and information deficits e.g. about good farming practices, the benefits of using improved seeds, market prices for their outputs, etc. Most of these problems are associated with the lack of knowledge and unavailability of timely and correct information to guide farmers. These knowledge and information deficits act as a critical constraint on achieving improvements in smallholder farmers' productivity and economic growth.

\subsection{FAABB framework and its components}

The FAABB framework is viewed as an integrated system that represents whole ARD related data, models, tools and platforms relevant to the monitoring and evaluation of farmers' performance, profitability, and economic and social welfare. The following six components, widely recognised as the 
basic components, define the proposed FAABB framework (Figure 1) for developing ARD m-apps
[13]. The framework components have been thoroughly analysed in [14] and other literature.

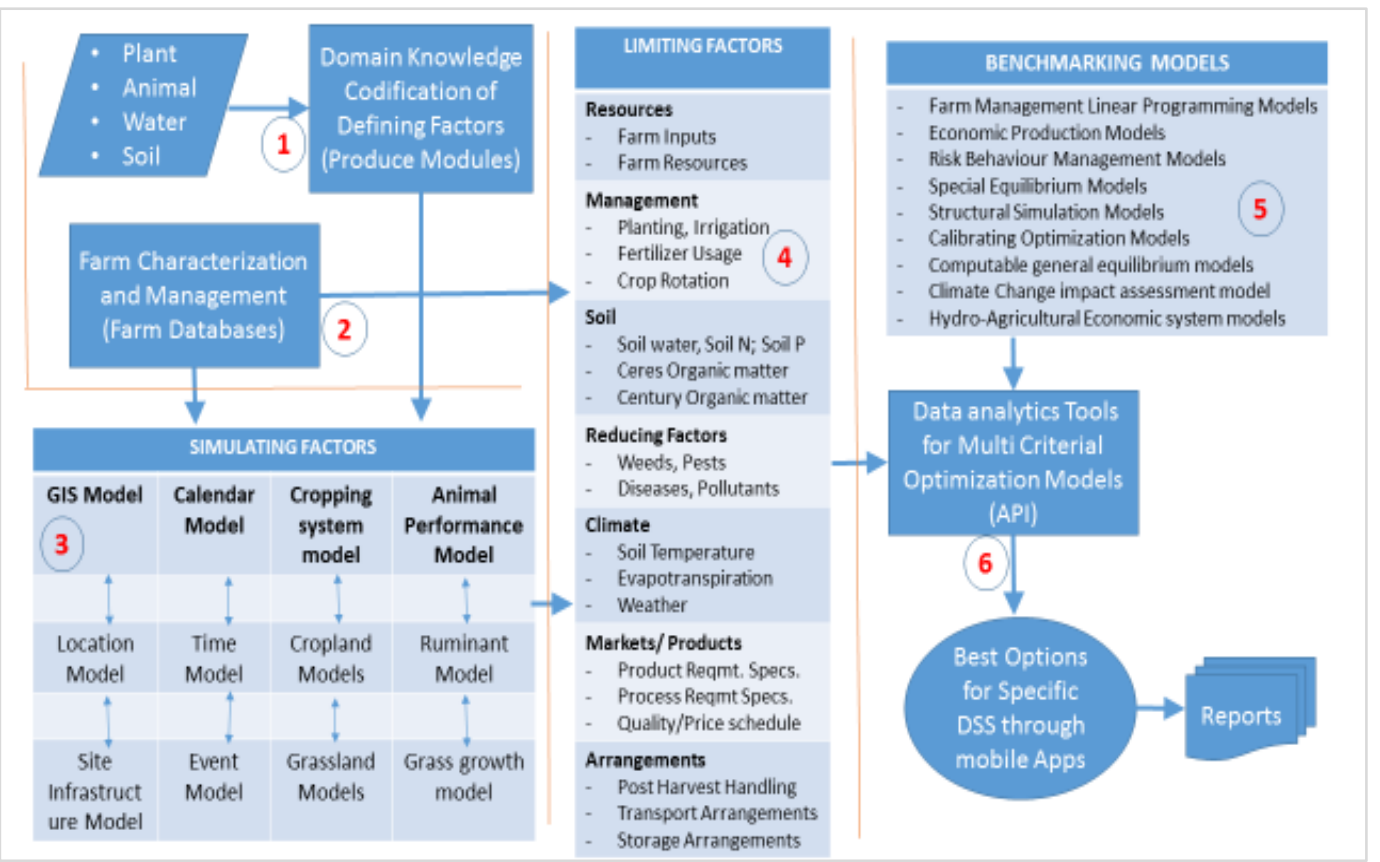

Figure 1 The FAABB Framework for developing ARD m-apps (Adapted from Herrero et al., 1996)

[Source: Herrero MT, Fawcett RH, Dent JB. Integrating simulation models to optimise nutrition and management for dairy farms: a methodology. Wageningen Pers.]

Defining Factors: The defining factors in agriculture are intended to codify the characteristics of the target produces. These factors predict crop/herd growth and yield at farm level and are typically obtained by narrowing down the many factors that are needed to estimate full potential production [15].

Farm Characterisation: Potential production models also include capturing data regarding the farm's specific characteristics that qualify specific produce in a specific farm location. Farm characterization also captures alternate crop rotations and product dependences for higher farm productivity/gains. Simulated Factors: In some cases, the complexity of farming systems justifies use of optimization tools to simulate different factors and their orientation (that would otherwise be complex to prescribe by practitioners) [16].

Limiting Factors: Limiting factors are formalized as constraints on the defining factors and restrict the farm from reaching full potential. For example: water-limited and/or nutrient-limited production constrains the defining factors for achieving full growth potential.
Other limiting factors include farm resource availability, climate, market requirements, logistics, etc. [13].

Benchmarking Factors: These are tools that formalise predetermined benchmarking models to predict the actual outcomes of the farm's parameters in the presence of constraints provided by the limiting factors. The resource allocation, performance management, certification, economic profitability, farm selection, etc. are also predicted through benchmarking modelling [11].

Data analytics: These are decision support tools, guided by specific use-cases that take the outcome of one or more benchmarking results and produce tailor made reports (aimed at guiding decision makers, informing farmers, achieving selections, managing change, etc.) [17].

The FAABB cyber-studio is intended to build a framework that will allow mobile and/or web-based application developers to define scenarios and usecases; and generate useful m-apps by tapping into data warehouse and libraries of reusable components 
for agricultural setups in Tanzania (to be maintained at the NM-AIST).

\subsection{Existing FAABB m-apps development frameworks}

Reference [9] testifies that mobile handsets are widely used for various purposes including FAABB. Typically, m-apps for FAABB could provide the most economical, practical, and social information to various stakeholders, especially smallholder farmers that are currently excluded from their utility.

Reference [18] summarizes 15 framework case studies from three countries, which have partial utility for FAABB. The study provides eight critical FAABB application areas, namely: (1) Price information (2) Market links (3) Extension and support (4) Distribution, logistics, \& traceability (5) Resource management (6) Labour migration \& human development (7) Governance/political issues, and (8) Rural finance infrastructure. None of the existing m-apps has more than three FAABB tools mentioned above. More critical is that no single application links market access, extension services and resource management within a single application. This suggests that none of the existing applications have an embedded framework for realising FAABB through mobile apps.

\section{Materials and methods}

As a direct implication of issues reported in the previous sections, two critical challenges emerge that make the existing frameworks and models less utilizable. First, is the knowledge codification problem. There is no single ARD system design framework that systematically captures the knowledge that comes out of various models and codifies them for future adaptation and/or use for generating new decision support systems (DSS) for specific use-cases (m-apps). Second, is the challenge of having software development toolkits (SDKs) with Application Programming Interfaces (APIs) that are generic and ubiquitous enough to facilitate the development of $\mathrm{m}$-apps to support a wide range of use-cases.

In order to address the above two critical challenges, the information architecture was developed at NMAIST, Arusha campus, Tanzania to simulate the platform to facilitate the development of mobile applications for FAABB. This section summarises the architectural design of the FAABB framework from three views: the network architecture, the information architecture, and the API.

\subsection{Enterprise architecture for FAABB m-apps development at NM-AIST}

Figure 2 provides a framework architecture for the interaction between various stakeholders involved in the knowledge codification process within the southern agricultural growth corridor of Tanzania (SAGCOT). The main data collection agent for FAABB is the extension officer (EO) through his/her mobile phone.

As indicated in the figure, there are two routes through which farm data is captured. The first route is through a centralised set of mobile application tools that are developed at NM-AIST and made available to EOs through their mobile phones. Each EO is assigned a group of farmers in the system and all of their data and reports are stored directly in the NM-AIST servers as internal data and tagged with specific dates. Various sets of internal data collected over different farms and different time lines are aggregated through data warehouse. The second route is to collect farm data through other agents that interact with farmers (particularly the farmers' market providers and service providers). Specialized mobile apps are developed as connect-and-exchange interfaces that obtain specific use cases from the EO's mobile and trigger specific operations at the agent's system and generate use case reports that are further sent to NM-AIST and stored again as internal data.

Farm-level data is captured directly by the EO into the system as (s) he interacts with the farmers through "m-apps" loaded on their phones. The market-level data is captured by the off-takers and/or service providers who (in-turn) will push to or pull the data from the NM-AIST FAABB cyber studio through specialized "connect-and-exchange" interfaces at their office terminals. Various stakeholders could then have access to the reports that come out of the system either through their phones or web portals to provide them with specialised reports. 


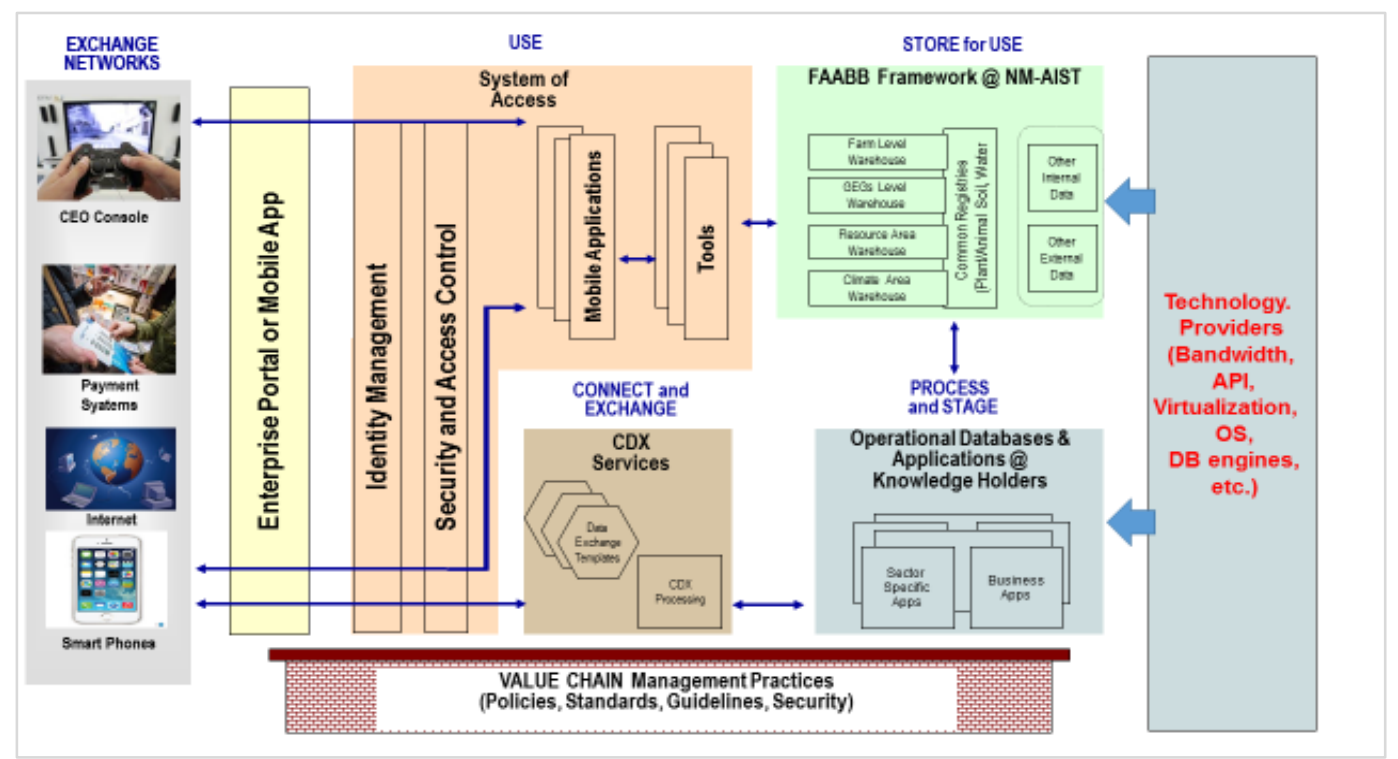

Figure 2 The enterprise architecture and its orientation to support FAABB framework at NM-AIST

The main principles behind the NM-AIST enterprise architecture for the FAABB are the following:

1. Farmers can be registered in the system at NMAIST electronically through mobile devices.

2. Farm characterization and management system for FAABB can be managed from a mobile device.

3. Off-takers with their own IT systems can connect to NM-AIST serves through dedicated connect and exchange interfaces otherwise the NM-AIST system suffices as a tool for electronic DSS through mobile devices.

Infrastructure as a service for mobile app developers was designed at NM-AIST to provide both Computing Resources as well as Networking Resources.

Infrastructure resources include:

- Storage services provide for durable and highly available and scalable cloud storage service

- Blob storage services provide for REST-based object storage for unstructured data

- Queue storage for upscaling services based on the needs

- Hosting for mobile app backends

- A notification service to provide for sending push notifications, SMS, or Email notification from any backend system.

- API management to publish API's for developers, partners at scale and securely
Networking Resources include:

- Virtual networking tools to allow for the provision of private networks and optionally connect to onpremise datacenter/computer.

- DDoS protection to allow for protection against distributed denial of service attacks

- Application gateways to guarantee protection from common web vulnerabilities and exploits like SQL Injection or cross site scripting.

- Load balancer to allow for automatically scales with increasing application traffic without the need to reconfigure or manage the load balancer.

- Traffic management.

The above architecture was validated and endorsed through the agricultural public private partnership (PPP) initiative, coordinated by the SAGCOT and involving the Government of Tanzania coordinated through the Prime Minister's Office, the Development partners, the local Private sector, the international private sector, and associations of smallholder farmers in Tanzania (ref. www.sagcotctf.co.tz).

\subsection{The information architecture for FAABB m- apps Development}

The information architecture is a four-layered system in that it cohesively connects all envisaged model concepts through defining factors, limiting factors, and benchmarking factors. It is the heart of the envisaged FAABB cyber studio at NM-AIST, as illustrated in Figure 3. 


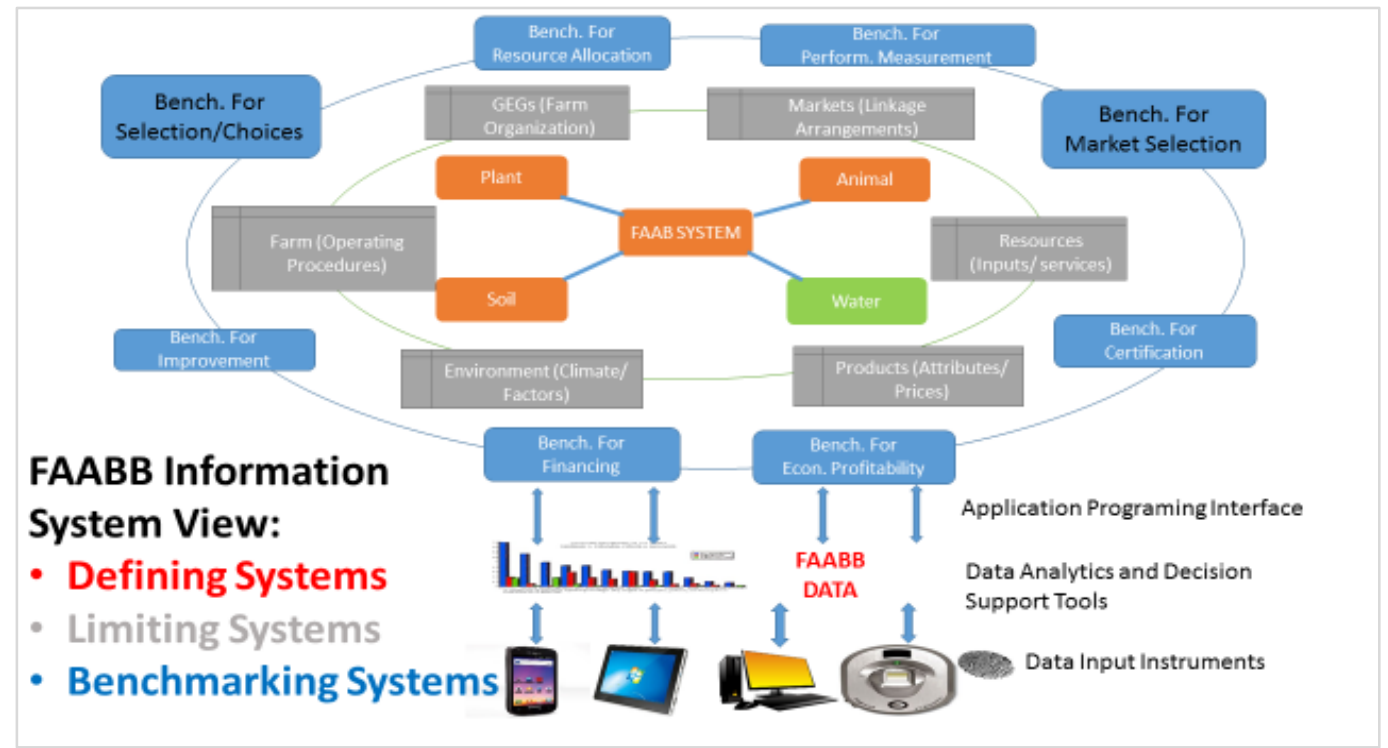

Figure 3 A framework architecture for codifying FAABB knowledge

The first inner layer is the "Farm Characterization Systems" layer that captures and presents the echo system set up for enabling specific farm characterization and management. It captures all relevant context-driven data to serve a specific instance of the FAABB at specific locations. It is driven by the principle of location (i.e. "no farms can be defined without location" principle).

The second inner layer is the "Defining Systems" layer that codifies the basic types of agricultural production variables and defines performance benchmarks/KPIs for each of the four dependent variables (i.e. soil, crop, animal and water). The modules that drive this layer provides a codified knowledge of "How-to guides" that describe how production processes should be conducted on the farm in the absence of constraints. The "How To" guides are typically adapted from a country context and made available to the public in the database context in a form of codified rules or events. This framework layer is envisaged to codify a Tanzanian baseline data for soil, plants, animals, and water in specific locations. Each defining factor should be associated with a location data in the first layer before its KPI is defined.

The third inner layer is the "Limiting Systems" layer that captures the possible contextual factors that may limit the attainment of the KPIs in the second layer. The limiting factors are either a result of natural environmental setups or available technologies that allow for accessibility of environmental information (mainly through simulations).

The fourth outer layer is the "Benchmarking Systems" layer that performs analytics for the purposes of providing DSSs. Theoretically, Each of the DSS is a function or a combination of the parameters of the previous three layers passed through a chosen modelling platform that structures the validity and visibility of information to the intended user. In situations where the output is intended to be m-apps, the application development toolkit (ADT) will further be required to pull and push information to and from the mobile user and the DSS. Other instruments other than mobile devices can also be used. Information Architecture as a service to mobile app developers has been designed at NM-AIST to provide both Database engine resources, database development tools as well as Data analytics services.

Database engine resources include:

- NoSQL database services through Mongo DB

- Big data services to support big data including data replication across multiple systems

- SQL database services through MS SQL

Database development tools include:

- Relations services for managing relations

- Cache services for high throughput, low latency data access

- Automated backup services

- Distributed, multi-model database at any scale 
Data Analytics Services include:

- Platforms to gather, store, process, analyse, and visualize data of any variety, volume, or velocity.

- Data modelling services.

- Data end point visualization services

- Data visualization services.

The information architecture for FAAB has been validated through three pilot projects in the SAGCOT regions involving two off-takers for dairy farmers (engaging over 2000 dairy farmers) and one off-taker for crop farmers (involving over 500 paddy/rice farmers).

2.3API to support m-apps development through the FAABB cyber studio at NM-AIST

A critical path for making FAABB Framework usable was the creation of FAABB API to support the m-app developers. The FAABB architecture allows API providers to deliver data in multiple formats such as plain text, HTML, XML, YAML, and JSON, which is one of its most loved features. In this section, we provide a few sample calls that have been developed to make up the FAABB API.

FAABB API Call: Query single farmer by ID Example JSON Data

\{"id": 343,"created_at": "2018-07-13

15:21:29","updated_at": "2018-07-13

15:21:53","first_name": "blayson","middle_name":

"n/a","sur_name": "milinga","gender":

"Male","date_of_birth": "n/A","phone_number":

"n/a","email_address": "n/a","postal_address":

"n/a","physical_address": "n/a","house_size":

"6","marital_status": "TBD","region_id":

"34","district_id": "140","ward_id":

"306","village_id": "12","group_id":

"206","registration_date":

null,"group_registration_number": null,"crop_id":

"2","farmer_id": "34140306122065266","land_size": "37","soil_condition": "N/A","latitude":

"N/A","longitude": "N/A","user_id": null,"lead_id": null\}

'/prototype/api/farmers/farmer/create', POST

\section{FAABB API Call: Create Farmer}

Example JSON Data

\{first_name": "blayson","middle_name":

"n/a","sur_name": "milinga","gender":

"Male","date_of_birth": "n/A","phone_number":

"n/a","email_address": "n/a","postal_address":

"n/a","physical_address": "n/a","house_size":

"6","marital_status": "TBD","region_id":

"34","district_id": "140","ward_id": "306","village_id": "12","group_id":

"206","registration_date":

null,"group_registration_number": null,"crop_id":

"2","farmer_id": "34140306122065266","land_size": "37","soil_condition": "N/A","latitude":

"N/A","longitude": "N/A","user_id": null,"lead_id": null\}

'/prototype/api/farmers/farmer/delete/\{id\}', DELETE Delete Farmer by ID

'/prototype/api/farmers/farmer/edit/\{id\}', PUT

\section{FAABB API Call: Edit Farmer by ID}

Example JSON Data

\{"id": 343,"created_at": "2018-07-13

15:21:29","updated_at": "2018-07-13

15:21:53","first_name": "blayson","middle_name":

"n/a","sur_name": "milinga","gender":

"Male","date_of_birth": "n/A","phone_number":

"n/a","email_address": "n/a","postal_address":

"n/a","physical_address": "n/a","house_size":

"6","marital_status": "TBD","region_id":

"34","district_id": "140","ward_id":

"306","village_id": "12","group_id":

"206","registration_date":

null,"group_registration_number": null,"crop_id":

"2","farmer_id": "34140306122065266","land_size":

"37","soil_condition": "N/A","latitude":

"N/A","longitude": "N/A","user_id": null,"lead_id": null\}

'/prototype/api/farmers/farmer/add/land_details/\{id \}', POST

FAABB API Call: Update land details by ID Example JSON Data

\{“land_size ": "10","longitude": "n/a","latitude":

"n/a"," soil_condition ": "n/a\}

\section{FAABB API Call: Return}

Example JSON Data \{"id": 343,"created_at": "2018-

07-13 15:21:29","updated_at": "2018-07-13

15:21:53","first_name": "blayson","middle_name":

"n/a","sur_name": "milinga","gender":

"Male","date_of_birth": "n/A","phone_number":

"n/a","email_address": "n/a","postal_address":

"n/a","physical_address": "n/a","house_size":

"6","marital_status": "TBD","region_id":

"34","district_id": "140","ward_id":

"306","village_id": "12","group_id":

"206","registration_date":

null,"group_registration_number": null,"crop_id":

"2","farmer_id": "34140306122065266","land_size":

"37","soil_condition": "N/A","latitude":

"N/A","longitude": "N/A","user_id": null,"lead_id":

null\} 
'/prototype/api/farmers/farmer/search', POST

\section{FAABB API Call: Returns}

Example JSON Data

[\{"id": 343,"created_at": "2018-07-13

15:21:29","updated_at": "2018-07-13

15:21:53","first_name": "blayson","middle_name":

"n/a","sur_name": "milinga","gender":

"Male","date_of_birth": "n/A","phone_number":

"n/a","email_address": "n/a","postal_address":

"n/a","physical_address": "n/a","house_size":

"6","marital_status": "TBD","region_id":

"34",,district_id": "140",,ward_id": "306

","village_id": "12","group_id":

"206","registration_date":

null,"group_registration_number": null,"crop_id":

"2","farmer_id": "34140306122065266","land_size":

"37","soil_condition": "N/A","latitude":

"N/A","longitude": "N/A","user_id": null,"lead_id":

null\}]

Group Module

'/prototype/api/groups', GET

\section{FAABB API Call: Get group by ID}

\section{Example JSON Data}

\{"id": 13,"created_at": "2018-08-05

12:42:57","updated_at": "2018-08-05

12:42:57","name":

"Twiyendage","registration_number":

"IDC/PTC/34","registration_date": "2016-01-

01","chairperson": "Peter Mbata","phone_number":

"+255753770658","info": "The group do not have

bank account","region_id": "14","email":

"Mbata@gmail.com","district_id": "35","ward_id":

"434","village_id": "94","secretary": "Aidan

sanga","secretary_phone_number":

"+255762907811","secretary_email":

"Sanga@gmail.com","accountant": "Gelefasi

kenza","accountant_phone_number":

"+2555765123343","accountant_email":

"Kenza@gmail.com"\}

'/prototype/api/groups/group/create', POST

\section{FAABB API Call: Create group}

Example JSON Data

\{"id": 13,"created_at": "2018-08-05

12:42:57","updated_at": "2018-08-05

12:42:57","name":

"Twiyendage","registration_number":

"IDC/PTC/34","registration_date": "2016-01-

01","chairperson": "Peter Mbata","phone_number":

"+255753770658","info": "The group do not have

bank account","region_id": "14","email":

"Mbata@gmail.com","district_id": "35","ward_id": "434","village_id": "94","secretary": "Aidan

sanga","secretary_phone_number":

"+255762907811","secretary_email":

"Sanga@gmail.com","accountant": "Gelefasi

kenza","accountant_phone_number":

"+2555765123343","accountant_email":

"Kenza@gmail.com"\}

'/prototype/api/groups/group/edit/\{id $\}$ ', PUT

Other calls have been (and are being) developed as part of the ongoing work at the NM-AIST FAABB Studio to support more use cases for future m-apps developers.

\section{Results}

This section presents snapshots of the three benchmarking reports that were produced from our FAABB system at NM-AIST during interactions with extension officers' mobile phones to aid the discussions and choices with the rural crop farmers in the SAGCOT area in Tanzania. The reports are consistently configured to present data in four different formats. On top of each report is a summary information for the population within the parameters that are chosen for the analysis. At the bottom left the report presents a map of Tanzania indicating the location(s) that are associated with the population under investigation. The semi-circled graph on the left presents the selected parameter values relative to the population values. The detailed benchmarking analysis is presented in three different views: the tabular view on the upper right corner, the bar chart in the middle of the report, and pie charts at the bottom left corner. In this way it becomes easy for various stakeholders to interpret the FAABB results consistently.

\subsection{Benchmarking for farm selection}

It is important to identify farmers in the learning group or in the area who are performing well and can be regarded as benchmarks. With technical guidance from the extension worker, farmers should agree on the farm or farms to be used. It is also important that those are considered representative of a known farm type, so that those conclusions drawn would have the widest possible application. In some cases, it will be possible to use the off-takers required number as the benchmark. A critical outcome of this process is to document and prescribe the basic data elements and their average values. Figure 4 presents a report of potential maize farmers in Tanzania. 


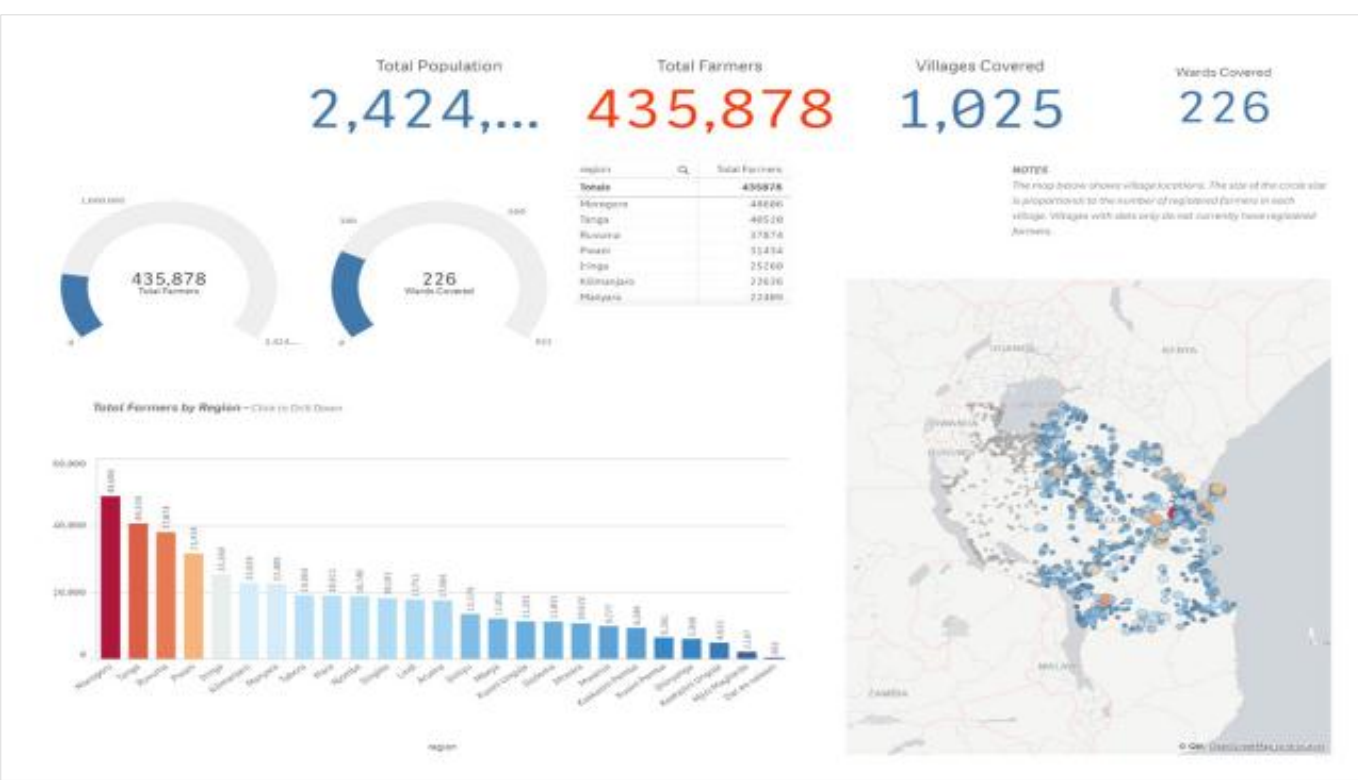

Figure 4 FAABB report for selecting the benchmark maize farms for SAGCOT

As indicated in the report, the map shows data from villages in the entire country where farmers are involved in growing maize. In the map, the size of the circle is proportional to the number of farmers who grow maize. Villages with small grey dots have potential for growing maize, but are currently not registered in the system. They could form part of the benchmarking exercise once they become registered in the future. Both the table at the top right corner and the bar chart at the bottom left corner indicates the best seven regions with high number of maize farmers. These regions exceeded the benchmark of having at least 20,000 farmers involved in maize business. From this report, it was easy for the banks and donors to invest in the four regions of SAGCOT that passed the benchmarks (i.e. Morogoro, Pwani, Ruvuma, and Iringa regions).

\subsection{Benchmarking for GEGs performance monitoring}

Comparisons of the performance of the smallholder farmer's business with the benchmark farm are made at group-farm level as opposed to individual farmers. Sales and food security indicators were used to analyse performance of GEGs in SAGCOT and identify gaps. These gaps can suggest weaknesses within the farming system and the reasons for them. Once areas of improvement have been identified, it is useful to compare them with benchmark farms in more detail. Digging beneath the data will help to understand why a particular farmer in the GEG is not doing better than the farmers in the benchmark group. The EO's smart phone was used to retrieve various pre-configured reports for the Songea rural district in SAGCOT. As indicated in Figure 5, the red circle on the map indicates the location of Songea rural as the focus of analysis. The top line provides global statistics of maize production in Songea rural and the number of farmers involved.

As indicated in the report, the acreage covered in maize is only 11,620 compared to the total available acreage of 34,767 acres in the Songea rural district. The report further indicates that only seven villages contribute to the high number of sales and have the highest reserves that contribute to food security. This report could trigger more analysis to find out why these villages were performing better than the rest of the village which are seemingly subjected to the same climate. 


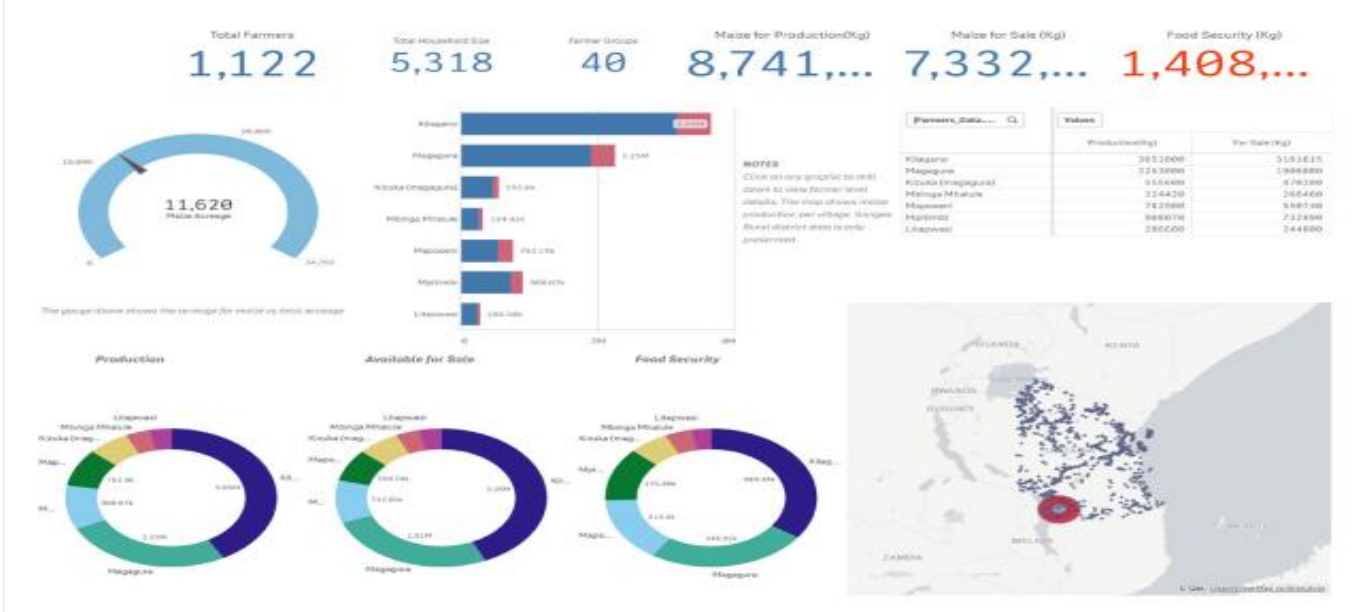

Figure 5 FAABB report for understanding the performance of GEGs in maize production

\subsection{Benchmarking for change management}

The purpose of identifying performance gaps and causes is ultimately to introduce actions and devise plans that the farmers in the GEG can use to improve the performance of their farms. Plans should include realistic targets for each farmer to achieve. EO can encourage, and support farmers and guide them in action planning through automated case studies retrieved through a smart phone. As indicated in Figure 6, the population covers only one GEG. The report shows that only 9 out of 25 members of the GEG contributed to half of the GEG's total production and half of its sales. The analysis shows further that the same nine members contributed almost $70 \%$ of the food security requirements for the GEG.
This benchmarking report provides the basic insights for addressing the problem of what is wrong with the rest of the members in the same GEG. Since the FAABB system provides for zoom-in reports, it was easy to click on the individual farmer and produce a more detailed report of the farm resource utilization. In this way, the EO was able to use the system reports in guiding discussions with farmers in the GEG.

As noted here, in the absence of benchmarking analysis, it was also difficult to convince the farmers to change. Most of the farmers in rural areas rely mostly on their traditional belief and unless one comes with such evidence-based analysis, it becomes difficult to manage change.

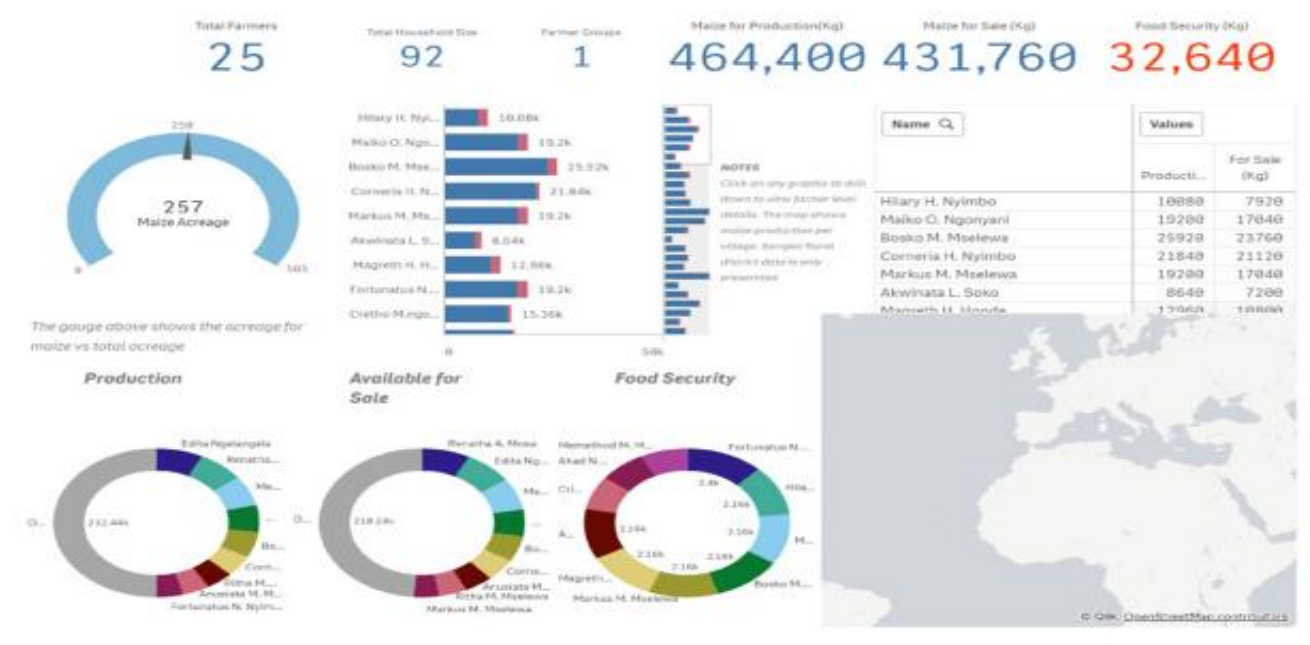

Figure 6 FAABB report for managing change within GEG member farms 


\section{Discussion on the framework utility in SAGCOT}

The NM-AIST cyber studio was evaluated for its utility to assist various SAGCOT stakeholders engaged in FAABB. Typical FAABB use-cases could be replicated in other environments aimed at addressing the FAAB challenges through similar benchmarking and DSS. With backing from the Government of Tanzania, development partners, and the private sector, NM-AIST has taken the upfront costs of developing a platform for the FAABB realization framework (see Figure 7) that will serve the corridor (as a pilot).

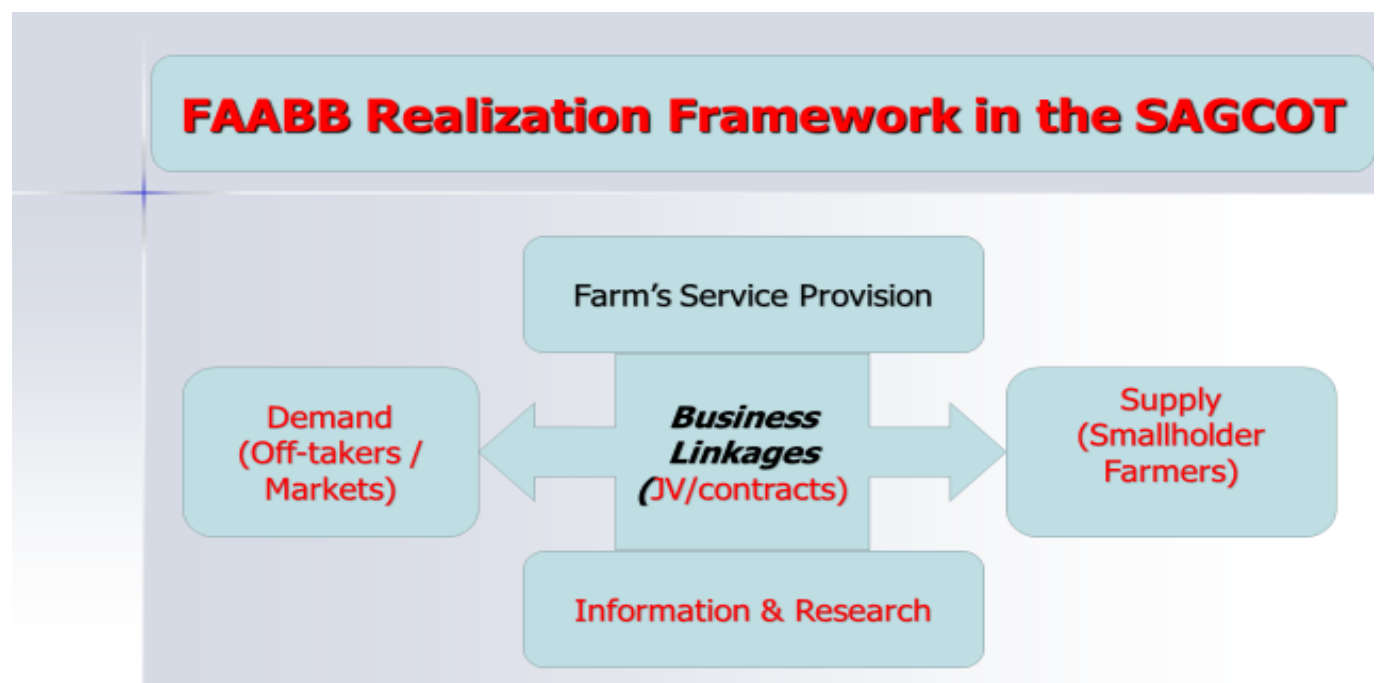

Figure 7 The business model for supporting FAABB Framework

This section highlights the five uses cases through which the system has been tested to justify the longterm investment in the proposed FAABB Framework realised through the cyber studio at NM-AIST.

\subsection{Facilitating extension services in the SAGCOT} areas

In this use-case, an extension officer (EO) is providing advice to a group of paddy farmers in SAGCOT. There is a need to help the EO register individual farmers in the group, manage their expectations in terms of required farm services and how to apply them to attain expected yields, and monitor their performance as they participate in FAABB [3].

Existing stems in SAGCOT are rarely connected to any database for identifying and authenticating farmers. Information on farmers ownership of their land is not easily available, leave alone codification of their boundaries through GPS and land management systems. While most of the data in SAGCOT are obtained through farmers' market providers, the correctness of information in existing systems on market providers become questionable as they allow data to be entered by hand. In some instances, EOs do not visit farms, but fabricate data since there are no tools that authenticate EOs presence and positions on respective sites. Early testing of the FAABB system at NM-AIST has shown success.

4.2Optimizing agricultural practice in the SAGCOT through DSSs

Government ministries responsible for agriculture possess information on agricultural best practices as well as weather information. Their challenge is often reaching the farmer in the last mile, or continuously updating extension officers with the latest information. The insufficient number of experienced extension officers and farm managers engaged in agriculture sector in general [5] and the crops [12], fishing [19], and livestock [6] subsectors in particular, in part, reflect the reality that farm management practices add on to the compounded problem farmers face in achieving FAABB. By providing these stored/forecast data as free public good and supporting information service delivery through the FAABB cyber studio at NM-AIST, many more farmers can benefit from these agronomical insights. A typical purpose of the DSS in SAGCOT areas is to record and benchmark farm economic performance of dairy herds of all sizes, in order to identify the top $25 \%$ of the industry within each 
management system, and to drill into why they are outperforming their peers, confirming critical best practices. The performance data analysis identifies the elite animals in the upper quartile to assist farmers with selection decisions (breed and strain) and to develop and refine a local profitability index for cows, cow families and the future local bull team. The DSS that has at least three sub modules for each of Soil, Crop and Animals should include a series of "How To" guides that describe how processes are conducted on farm in simple or localised languages. Over time these guides will enable efficient monitoring and management of extension officer service to farmers and reinforces "FAABB" thinking and habits for farmers. We are currently applying the NM-AIST system to document the Swahili language mnemonics for the reports that come out of the system.

\subsection{Stimulating rural finance in SAGCOT}

Smallholder farmers in the SAGCOT area often not informed about the opportunities for accessing loans from financial institutions. This is partly because of English language barriers, but even more so because of their income levels they cannot afford to travel from rural areas to the bank branches in the urban areas. The mobile based access to financial data and mobile payment services can help to overcome this problem. Of recent trend, more agents are investing in rural finance on the basis of loyalty programs that are driven by data about the farmers and their associated farm productivity indices [3].

Although there are m-apps that simulate loyalty programs as their basic benchmarking tools for lending to smallholder farmers, most of them are still not able to use farmers' FAABB compliance information as a basis for guaranteeing their loans. Instead, they are still required collaterals from farmers as a primary basis for managing their financial risks. Benchmarking for financial compliance requires DSSs with embedded models that integrate farmers' economic performance with farm productivity and efficiency. This is also a critical ongoing testing of the system which has shown successes.

\subsection{Facilitating the SAGCOT agribusiness value chain}

The demand side in one part of the value chain needs to know about the supply side providers in other parts of the value chain in order to decide who to engage. The necessary information to aid decision making include products and their required attributes, the inputs and their levels of application, the logistical arrangements and prices, the local government procedures and required taxes, etc. Although there are models that simulate logistical services like "Uber or Twende-Abiria" that use Google-maps to simulate the passenger's roots on mobile phones, these apps have not been able to serve the agribusiness sector because they require codification of services and products available by different actors on the value chain. Furthermore, these actors and reactors require a platform that allows reception of service enquiry and manage multiple subscribers to publish their offers.

The tools should provide links into prescribed local and international search engines and websites as credible sources of information that may be required by various actors for a given value chain. On the other hand, such tools may be connected to local radio and EOs network within geographical areas that could provide responses through voice or video clips. Our pilot has shown some good results through the system.

4.5Transparency and efficiency in enforcing agricultural policies in Tanzania

Many policy decisions in the government or parliament in Tanzania are permitted, restricted or forbidden based on estimated rather than actual data. Most of the time these decisions are based on practitioner's experience as opposed to the actual reality on the ground. The allocation of the budget for subsidizing farmers in Tanzania largely depends on generic (sometimes unrealistic) computations as opposed to adopting models that embed such information as the: locations/zones, types of crops, types of inputs, the number available farmers that are ready to cultivate a typical crop for the coming seasons, their purchasing power, etc.

Relevant datasets that can be available include land registration, licensed organisations (farmer groups), farm input requirements, import/export tariffs, and permitted pesticides, etc. The building of datawarehouse at NM-AIST is part and parcel of the ongoing FAABB framework to codify all existing and new policies that affect agribusinesses.

\subsection{Limitations of this work}

It should be acknowledged that this research work is in its infancy and will require more case studies and tests in the field before its utility is optimised and realised in solving the real challenges faced by ARD. More m-apps will have to be developed and validated 
based on this framework. More data will also have to be collected and stored at NM-AIST to provide a wider coverage of the whole country. We also need to embrace the "Internet-of-Things" (IoT) technology as the best way to collect simulated data and/or build interfaces into third party systems to pull data that has already been validated into the FAABB framework.

\section{Conclusion}

A review of the current state of mobile application development frameworks, shows that there has been less focus on their capabilities and limitations to support FAABB. Although the FAABB components are well known from the literature point of view, there has been no single framework that has acknowledged benchmarking as a crucial component of the farming business. The paper has presented a new framework to support m-apps development framework to support FAABB in the Tanzania context. The framework is implemented through a cyber Studio at the NM-AIST. The framework promises to address not only the knowledge codification problem, but also the need for a cultural change among agricultural researchers to ensure that data for addressing the range of use-cases are available for future mobile application development. The FAABB framework has been tested in the SAGCOT and its initial results provide a useful starting point for developing m-apps for targeted FAABB challenges in developing countries.

As a direct result of this work, the FAABB cyber studio was launched at NM-AIST in 2018. It is planned to extend this work by building on this initial version of the FAABB Framework by deepening the testing of the utility of the framework in more locations and through other product types. Future research is also intended to test the effectiveness of various models to FAABB.

Authors are invited policymakers, agriculture specialists and practitioners to join a FAABB cyber studio to add own contributions on the current version of the FAABB framework architecture for mapps development. Contributions could come from mathematical modelling, m-apps development, technology options, agronomical practices, data collection and data structures, big-data hosting, information security, etc.

\section{Acknowledgment}

This research was supported/partially supported by SAGCOT Catalytic Trust Fund. We thank our colleagues from Litenga Holdings (T) Ltd. and ABEA Ltd. who 377 provided platforms and technical expertise that greatly assisted the research, although they may not agree with all of the interpretations/conclusions of this paper. We would also like to show our gratitude to the staff and students of the School of Computational and Communication Sciences and Engineering (CoCSE) at NM-AIST for sharing their pearls of wisdom with us during the course of this research.

\section{Conflicts of interest}

The authors have no conflicts of interest to declare.

\section{References}

[1] Barham J, Chitemi C. Collective action initiatives to improve marketing performance: lessons from farmer groups in Tanzania. Food Policy. 2009; 34(1):53-9.

[2] Poulton C, Dorward A, Kydd J. The future of small farms: new directions for services, institutions, and intermediation. World Development. 2010; 38(10):1413-28.

[3] http://siteresources.worldbank.org/CSO/Resources/Tra nsformationalPartnershipstoStrengthenFoodSecurity FelixMosha.pdf. Accessed 12 May 2019.

[4] Wolfe ML, Ting KC, Scott N, Sharpley A, Jones JW, Verma L. Engineering solutions for food-energy-water systems: it is more than engineering. Journal of Environmental Studies and Sciences. 2016; 6(1):17282.

[5] Rwebangira N. Enhancing access to quality agriculture extension services for small holder farmers. Agricultural Non-State Actors Forum (ANSAF). 2017.

[6] Nell AJ, Schiere H, Bol S. Quick scan dairy sector Tanzania. Dutch Ministry of Economic Affairs; 2014.

[7] https://cgspace.cgiar.org/handle/10568/41910. Accessed 12 May 2019.

[8] Lwoga ET, Ngulube P, Stilwell C. Information needs and information seeking behaviour of small-scale farmers in Tanzania. Innovation: Journal of Appropriate Librarianship and Information Work in Southern Africa. 2010; 2010(40):82-103.

[9] A taxonomy of human computer interaction. Section 2 of the ACM SIGCHI Curricula for HCI. pp. 1-9, 1992. ACM Press.

[10] Mnenwa R, Maliti E. A comparative analysis of poverty incidence in farming systems of Tanzania. 2010.

[11] Kahan D. Farm business analysis using benchmarking. Food and Agriculture Organization of the United Nations; 2013.

[12] Belay K, Abebaw D. Challenges facing agricultural extension agents: a case study from south-western Ethiopia. African Development Review. 2004; 16(1):139-68.

[13] Van Ittersum MK, Cassman KG, Grassini P, Wolf J, Tittonell P, Hochman Z. Yield gap analysis with local to global relevance-a review. Field Crops Research. 2013; 143:4-17.

[14] Antle JM, Basso B, Conant RT, Godfray HC, Jones JW, Herrero $M$ et al. Towards a new generation of agricultural system data, models and knowledge 
products: design and improvement. Agricultural Systems. 2017; 155:255-68.

[15] De Wit A, Boogaard H, Fumagalli D, Janssen S, Knapen R, Van Kraalingen D, et al. 25 years of the WOFOST cropping systems model. Agricultural Systems. 2019; 168:154-67.

[16] Asai M, Moraine M, Ryschawy J, De Wit J, Hoshide AK, Martin G. Critical factors for crop-livestock integration beyond the farm level: a cross-analysis of worldwide case studies. Land Use Policy. 2018; 73:184-94.

[17] Pivoto D, Waquil PD, Talamini E, Finocchio CP, Dalla Corte VF, De Vargas Mores G. Scientific development of smart farming technologies and their application in Brazil. Information Processing in Agriculture. 2018; 5(1):21-32.

[18] Qiang CZ, Kuek SC, Dymond A, Esselaar S. Mobile applications for agriculture and rural development. ICT Sector Unit World Bank. 2012.

[19] Njera D, Chonde C, Kambewa D, Dzanja J, Kayambazinthu D, Kaunda E, et al. Member-specific characteristics affecting capacity of farmer organisations in promoting fish farming: case of Dowa and Mchinji districts in Malawi. International Journal of Research. 2016; 3(11): 6-12.

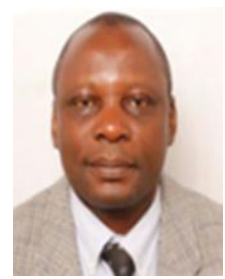

Mr. John Joel Kyaruzi is a $\mathrm{PhD}$ student at the Nelson Mandela African Institution of Science and Technology (NM-AIST) in Arusha, Tanzania. He is also the Executive Secretary of SAGCOT Catalytic Trust Fund. Formerly, he worked with the Tanzania Investment Centre (TIC) as the Director of Research and Information systems for 9 years, and a Lecturer in Computer Science at the University of Dar es Salaam for 15 years. He possesses MSc. degree in Computer Science from UNB, Canada. He also served as a Director of the African Virtual University at the University of Dar es Salaam for 4 years; He is also a champion of the Smart Card Systems for National IDs and other Payment Systems.

Email: kyaruzij@nm-aist.ac.tz

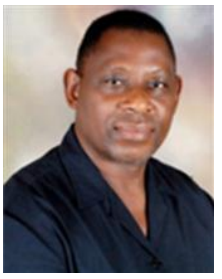

Eng. Dr. Zaipuna Obedi Yonah is a Senior Consulting Engineer in ICTs and Telecoms; and a Senior Researcher with Applied Engineering \& ByteWorks (T) Limited in Tanzania, also associated with the School of Computational and Communication Science and Engineering (COCSE) at NM-AIST. Eng. Dr. Zaipuna O. Yonah MIET, MIEEE holds a PhD (1994) Degrees in Computer-based Instrumentation and Control Engineering from the University of Saskatchewan, Saskatoon-Canada. In Tanzania, he is a Registered Consulting Engineer in ICTs. Dr. Yonah has over 30 years of practice. His work spans the academia, industry and policy making fields. He is one of the pioneers driving the national broadband agenda in Tanzania. His research interests include: ICT4D, Cyber Security, ICT Policy and Regulation, Mobile and Web Applications, High-Capacity Broadband Networks, and Intelligent Instrumentation and Control.

Email: zaipuna.yonah@aebw.co.tz

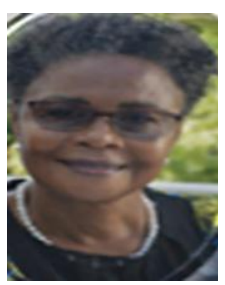

Prof. Hulda Shaidi Swai is a Professor in the School of Life Science and BioEngineering at the Nelson Mandela African Institution of Science and Technology (NM-AIST). Prof. Hulda Swai, a Nanotechnology Scientist, holds a $\mathrm{PhD}$ in Biomaterials, from Queen Mary's College, University of London, UK where she also worked as a Researcher for 9 years. Prior to joining the CSIR and NM-AIST, she worked as a Biomedical Materials Researcher at the Interdisciplinary Research Council (IRC), University of London, UK, in which she was part of a team that developed a polymer system which was used as a vehicle in the slow release delivery of anti-fungal drugs. This drug delivery system received Medical Control Agency approval for clinical trials in the UK for the HIV/AIDS-Candida infected patients. Through her exemplary research, she has been nominated for the Olusegun Obasanjo Prize for scientific discovery and/or technological innovation. In May 2018, Prof. Swai was appointed the President of the African Materials Research Society (AMRS) and in 2013 she was appointed as an extraordinary Professor at the University of Pretoria.

Email: hulda.swai@nm-aist.ac.tz 